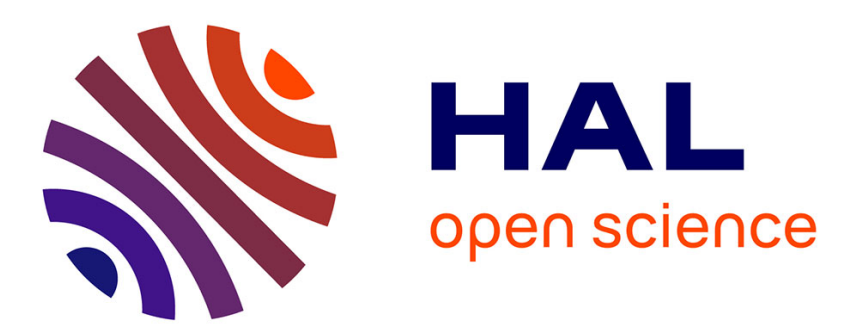

\title{
Towards Ontology-Aided Manufacturing and Supply Chain Management - Insights from a Foresight Research Stanislaw Strzelczak
}

\section{To cite this version:}

Stanislaw Strzelczak. Towards Ontology-Aided Manufacturing and Supply Chain Management Insights from a Foresight Research. IFIP International Conference on Advances in Production Management Systems (APMS), Sep 2015, Tokyo, Japan. pp.502-510, 10.1007/978-3-319-22759-7_58 . hal-01431138

\author{
HAL Id: hal-01431138 \\ https://hal.inria.fr/hal-01431138
}

Submitted on 10 Jan 2017

HAL is a multi-disciplinary open access archive for the deposit and dissemination of scientific research documents, whether they are published or not. The documents may come from teaching and research institutions in France or abroad, or from public or private research centers.
L'archive ouverte pluridisciplinaire HAL, est destinée au dépôt et à la diffusion de documents scientifiques de niveau recherche, publiés ou non, émanant des établissements d'enseignement et de recherche français ou étrangers, des laboratoires publics ou privés. 


\title{
Towards Ontology-Aided Manufacturing and Supply Chain Management - Insights from a Foresight Research
}

\author{
Stanisław Strzelczak \\ Warsaw University of Technology, Faculty of Production Engineering, Warsaw, Poland \\ s.strzelczak@wip.pw.edu.pl
}

\begin{abstract}
Ontologies attract growing interest as a mean for semantic integration of knowledge, applications, models and systems. This particularly applies to the ICT and automation intensive industries. The paper discusses possible novel ontology-aided solutions for managing manufacturing and logistics operations, which result from a foresight research of industries. The professionals from European companies along semi-structured interviewing and brainstorming provided a range of suggestions, mostly in terms of functional requirements. Some novel solutions have been derived and conceptualized this way.
\end{abstract}

Keywords. Ontology • Semantic integration • Manufacturing management • Supply chain management $\bullet$ Foresight research

\section{Introduction}

Manufacturing and logistics are recently exposed to the rapid growth of openness and networking. The outcome is increased complexity and variability of systems and processes. In parallel many innovations in information and communication technologies (ICT) and automation technologies (AT) are being implemented. Among the key enabling technologies there are: Web services, smart appliances, semantic technologies et al. The particular areas of ongoing and suggested development can be referred as the seven Internets [Fig.1]. The evolution of Internet towards the Semantic Web may eventually provide a core public infrastructure for information exchange for other Internets [1]. Some of them are advanced and undergo intensive development, like the social Internet or e-commerce. Other are still rather futuristic concepts than reality, e.g.: the Physical (logistical) Internet [2] or the Internet of Things and Systems. To note, many similar concepts are also discussed, like the Industrie 4.0 [3], which assumes future industrial systems to be built upon Cyber-Physical Systems (CPS) [4].

It is argued that major issues and challenges which may arise along development of seven Internets could be resolved by the means of ontology engineering [5,6,7]. It is considered as an enabler for novel systemic solutions and functionalities for manufacturing and supply chain management [8,9], which is the focus of this paper. However, it is argued that cross-organizational and externalized solutions require modified abstractions and formal extensions to enhance the capacities of existing ontologies [7]. 


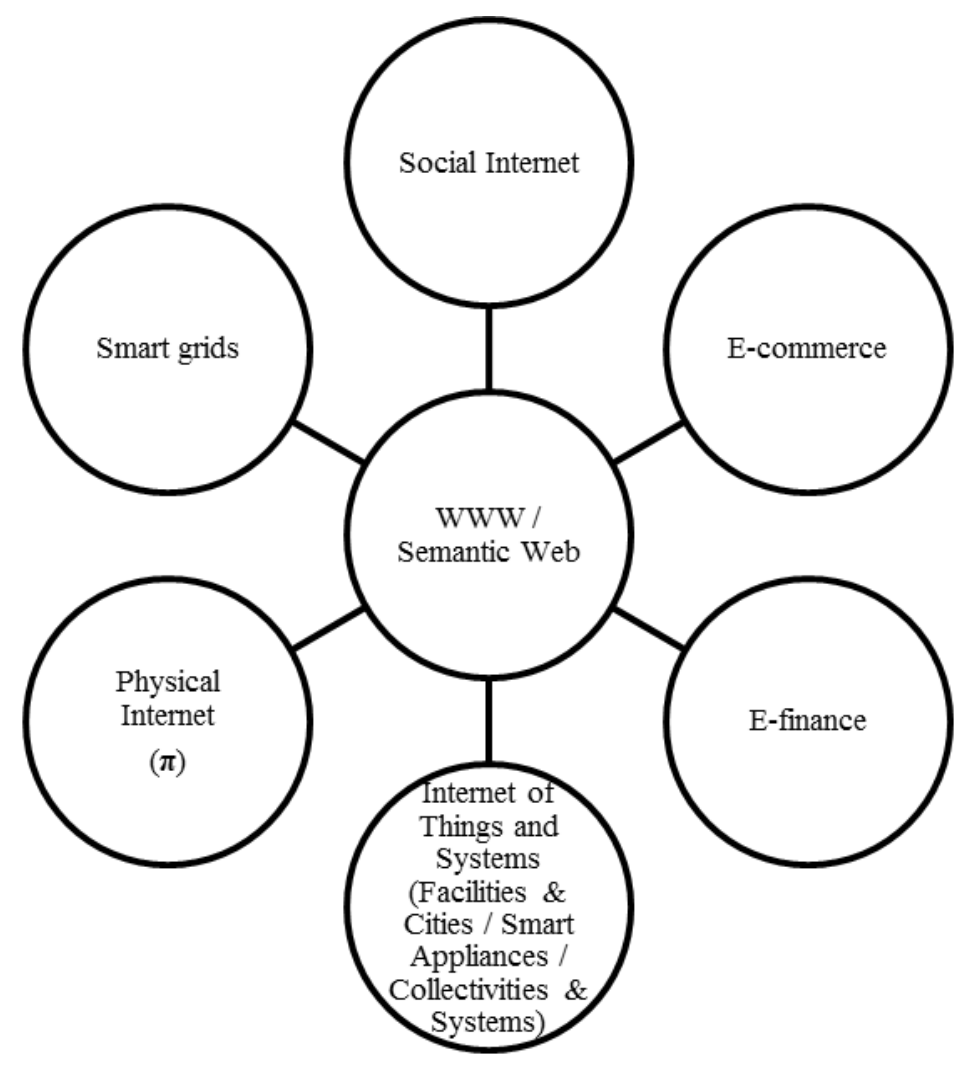

Fig. 1. Semantic Web of seven Internets

This paper presents and discusses results of a foresight research, which involved professionals from European industries. They were used to conceptualize a range of novel functionalities and systemic solutions for management of manufacturing and supply chain operations, which are presented in this paper.

\section{Methodology}

The research aims future needs and requirements, as seen by industries, with regard to the systemic solutions and functionalities for management of manufacturing and supply chains that could be eventually facilitated by the means of ontology engineering. From the available methods semi-structured interviewing of experts followed by brainstorming was chosen to protect dependability of results, to efficiently drive creative thinking, and to get high value-effort ratio along the research. It could not be presumed in advance that all professionals involved into the research have a competence in the ontology engineering. Therefore all of them have been equipped with a prior required knowledge of that kind. Obviously the experts were not expected to provide insights that could directly target detailed aspects of ontology engineering. 
Considering the purpose of research following categories of companies were identified as those having expertise in the area of interest:

1. Users of advanced ICT and AT technologies from discrete and process industries;

2. Providers of ICT and AT solutions (software, hardware);

3. Manufacturers of automated equipment;

4. Integrators of supply chains and manufacturing systems (ICT and AT solutions).

The following sectors were approached: automotive, petrochemical, chemical, cement and electro-power generation. The expertise from telecom sector on architectural and communication solutions was considered as a potential source of important insights. The structure of interviewed experts in terms of rank and respective type of expertise is presented below in Table 1 .

Table 1. Rank-based structure of interviewed experts

\begin{tabular}{|c|c|c|c|}
\hline No. & Rank of expert & Number & $\%$ \\
\hline 1 & CEOs & 4 & 5 \\
\hline 2 & CTOs & 5 & 7 \\
\hline 3 & Other $\mathrm{CxOs}$ & 1 & 1 \\
\hline 4 & $\Sigma \mathrm{CxOs}$ & 10 & 13 \\
\hline 5 & Middle level managers & 15 & 20 \\
\hline 6 & Engineers & 47 & 67 \\
\hline & 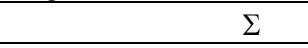 & 72 & - \\
\hline
\end{tabular}

Each company was approached by phone, then a formal letter was e-mailed, including a brief description of research and a list of guiding ideas on ontology engineering, and questions. Finally meetings were arranged at the company sites. The results of each interviewing were initially processed, than compiled and systemized. During the meetings questions provided in advance have been used to lead the interviews together with other direct, indirect, and ad hoc questions. This way many assessments, forecasts and recommendations could be obtained. Brainstorming was normally happening in case of some questions. All interviews were precisely documented, in most cases recorded. The questions provided in advance were tailored according to the company category and mostly focused around the following topics:

- New systemic solutions, in terms of structures, layouts, architectures, material and work flows, planning and control principles, patterns of serviced demand, etc.;

- New functionalities, including those driven by smart appliances, embedded appliances, and other novel ICT technologies, internally provided or outsourced;

- Other core novelties expected for open, knowledge-aided operations management; eventual impacts and outcomes from integration of seven Internets;

- Knowledge management; cognitive aspects; issues of utilization and maintenance;

- Various discrepancies and couplings, especially arising along processes;

- Human-machine interfaces (HMI) and human-systems interdependencies;

- Possible insights for developing foundations and means of ontology engineering;

- Business models and deployment patterns for new solutions. 
The variability of gathered opinions was compiled and synthesized. Controversial opinions were not ignored. Although the number of interviewees was moderate, using statistical analysis as a basis for justification of conclusions was assessed as of minor importance. Key systemic solutions and functionalities that were derived from the research results were then conceptualized as ideations. They are presented later on.

\section{$3 \quad$ Findings and conclusions}

The feedback received from experts did not differ much with regard to their hierarchical position. Not surprisingly the engineers exhibited a bit more interest in technical aspects, while the top level managers rather focused on systemic and functional aspects. The visible difference of interests and focus was linked to the type of industry, mainly with regard to the distinction of process and discrete industries.

Most interviewed experts recognize ontologies (or semantic expression of information) as an efficient representation and communication mean for open knowledgedriven manufacturing and logistics. In particular ontologies are expected to facilitate semantic integration of distributed smart (i.e. intelligent) applications, this way enabling various novel systemic solutions and functionalities.

Many interviewees named ontologies as generic vehicles for description of all conceptualizations and models to found applications used along operations management. This feature enables evolution of applications, following internal extensions and external provisions of knowledge and makes possible qualitatively new ways of communication and integration. Consequently ontologies are expected as enablers of new smart solutions for managing operations, which may self-adapt in a run-time mode. Therefore various changeabilities and novel functionalities can be also facilitated.

Concerning openness and interoperability in operations management, it was suggested that new concepts might be required to facilitate the novel solutions for operations management. Apart of 'material', or 'real' items, like facilities or all flowing items, virtual spatiotemporal items should be possibly conceptualized, considering mereotopological expressions, like: (i) virtual systems and capacities; (ii) spatiotemporal demand/task structures (projects, orders etc.); (iv) processes and flows; possibly all 'movable' or 'trackable' items could be subject of such considerations; (vi) spatiotemporal interdependencies concerning coordination patterns, e.g.: synchronizations, time-rigidness etc.; (vi) components of tacit knowledge.

Concerning planning and control principles and related functionalities, that could be a subject of knowledge-driven operations management, the following abilities are commonly recognized as most required and fundamental:

- Distributed / non-hierarchical / externalized / localized planning and control; e.g. by: self-management of subsystems or devices or orders; self-management of herds of devices or orders, e.g. through bargaining, auctioning and similar mechanisms;

- Ontology-aided planning and control, e.g.: bulking, issuing, accepting, initiating, prioritizing and dispatching orders, based on flexible processes/routings;

- Adaptive ongoing consideration of performance and operational targets;

- Adaptive ongoing consideration of conditioning and contextual factors. 
Following the above findings a range of other most required 'smart' abilities and functionalities was also suggested by the experts: (i) limited intelligence of embedded appliances, at least to support mostly requested self-adaptations to current local circumstances, e.g.: breakdowns, bottlenecks, blockings, starvations; (ii) capacities of local self-diagnostics and early-warning; (iii) aggregation of local data to support externally supported inferencing (e.g. from clouds, using Big Data technologies), like failures forecasting, optimized process control, setting standard times etc.; (iv) simple learning capacities, e.g. in reference to input-output control of manufacturing cells.

Direct communication between devices and other items (e.g. machine-to-schedule or truck-to-depot) together with the use of web based interfaces (appliances and users should be able to access Internet from any device or HMI, like: laptop, tablet, phone etc.), were commonly recognized as the basic for open communication and an enabler of some new functionalities.

Concerning functionalities to be eventually thrived from or built upon exploitation of tracking technologies, the following two were suggested as particularly important: (i) intelligent reporting/notifications about progressing processes or along forwarding; (ii) handling routings/routes in local memories of movable items; (iii) using creatively data about exact position; e.g. it could enable counting products in exact container or provide possibility to read a real-time position of each palette; each of those data could be stored and then analyzed in case of disruptions.

It must be underlined in reference to all above presented findings, that the experts from process industries exhibited a very focused pattern of interests. Automated process control and maintenance management are experienced as the two major concerns of plant management, while external logistics is in most cases considered a secondary issue, the following functionalities attracted the major attention of the experts: (i) adaptive or optimized process control, sometimes supported by distant links, even from different installations, which cannot be normally facilitated by existing massive and hierarchical control systems; these could help to reduce energy consumption, emissions, waste reduction etc.; (ii) extended diagnostic capacities, in particular early warning; these could much facilitate preventive maintenance and protect against outages and catastrophic events; (iii) improved monitoring abilities, also by HMIs and following the item (ii).

Interesting insights were provided by the telecom sector, which is highly advanced in research aiming at IP based integration of different technical equipment (not just of mobile phones), by means of cellular and other ICT technologies. The telecom experts suggested that smart phones can operate as networked embedded devices, being more efficient and cheaper than common comparable AT solutions. E.g. a collection of mobile phones can operate like a local network (at a site), in a peer-to-peer mode, using any interface/interfacing protocol and different modes of communication.

The telecom experts also recommend some technologies developed by their sector, which can eventually support process control under non-deterministic circumstances, particularly in case of failures or breakdowns of networked resources. For such purposes the Erlang open-source language has been developed, which targets programming of non-deterministic controls, e.g. with regard to flows in networks under unavailability of some resources. 
Some interviewed suggested applying an open source approach for production ontologies, at least for the core ontologies that could be publicly shared. The issue of who and how should maintain them was noticed. Other experts suggested impossibility to develop a unified core ontology for the domain. E.g. they suggested impossibility to handle unique control models, considering specific complexities of exact systems. Anyway, core shared ontologies were suggested as a probable realistic solution.

The above synthesis of findings, which were obtained along the foresight research, provides comprehensive and consistent guidelines for various ontology-aided innovations addressing operations management. They could be straightly used for conceptualizing some novel solutions and functionalities presented in the next section.

\section{Novel ideations for systemic solutions and functionalities}

This section presents some novel ontology-aided systemic solutions and functionalities, which could be developed for manufacturing and supply chain management. All of them were derived or conceptualized from the results of foresight research. Apart of enabling use of ontologies using some novel ICT and AT technologies was also considered. The ideations to some extent overlap. It is a minor issue, as their role is to help identifying directions for further R\&D. All of them may potentially provide significant advantages, in terms of functional capacities or performance, in relation to the existing solutions. They are realistic as all of them can be eventually implemented using the existing technologies, despite some requested R\&D. They are as follows:

- (A) Merging electronic markets with manufacturers and logistic providers (or Ecommerce + Production \& logistics Internet): Electronic markets could be integrated through Semantic Web services (e.g. clouds) with a pool of manufacturers and logistics providers (or logistics infrastructure, like the Physical internet). Different planning and/or coordination mechanisms could be exploited within such ecosystems, ranging from push-flows, through pull-flows, to limited ad-hoc markets (e.g. auctioning et al.). Demand, material and other flows could be adaptively encapsulated along operations, thus thriving from the transaction economy of scale.

- (B) Supply chain-wide operational alignment: Overall planning and control service could integrate operational management of demand and material flows. Various mechanisms could be exploited, depending on the characteristics of demand, products, processes, and particularly in reference to the couplings and discrepancies. They could range from centralized to distributed and local, like in case (A). Similarly, different encapsulated flows could be applied along operations. The transaction economy of scale and utilization of resources could be significantly improved this way. Distortion of demand could be reduced, like the turbulences of flows. This kind of solution could be applied as a dedicated one or like a public service.

- (C) Non-hierarchical and/or distributed management of operations: A manufacturing system or a supply chain or a logistical system could be operated mostly or solely by smart appliances, embedded or assigned to various components of the system, both physical \& virtual. E.g. demands (orders) could be managed and controlled by dedicated intelligent agents, who would solely supervise their execution 
(i.e. like self-manageable orders). The agents would apply for resources in a runtime mode. Similarly resources could be equipped with managing agents. The demand, work and material flows could be composed or adapted at a run-time. Relevant control mechanisms would be used by the agents, e.g. auctioning or prioritizing mechanisms, eventually supervision by operators or other users. Planning and/or coordinating agents could be involved in these activities. The above means that eventually flows and resources could somehow manage themselves.

- (D) Herd control of operations: Complex manufacturing and logistical demands could be distributed to various providers/vendors, then operating like a herd. Management and control could follow bio-mimetic and/or eco-mimetic imitations. Under some circumstances users (humans) could be subordinated to smart agents, who could actually control inter-organizational flows of demands or deliveries.

- (E) New functionalities: Due to advantages of Big Data technologies or using the power of cloud computing new functionalities could be added for occasional or run-time use. Alternatively the existing functionalities can be provided in a more efficient and functional way, using a limited intelligence. E.g.: monitoring of resources, process planning, monitoring of processes, adaptive control of flows etc.

- $(F)$ Adaptive management of operations: Modes of planning and controlling operations could be adapted to follow phase transitions of systems/collectivities and processes, or of the operational environment. E.g. dispatching rules in a manufacturing system could be changed according to the load or temporary bottlenecks, processes (routings) could be redefined in case of outages and jams, etc. Such types of functionalities are primarily enabled by the capacity of intelligence. It is worth to note, that anticipation of phase transitions can be significantly enhanced when considering extended environments, e.g. supply chain wide, and when exploiting Big Data.

The above ideations illustrate some novel solutions, which were conceptualized from the foresight research. All of them assume using ontologies for knowledge representation and communication, and for reasoning. This applies in particular to those aspects of operations, which refer to complexities. All ideations provide significant functional and performance advantages. They suggest directions for further R\&D.

\section{Summary and further research}

The foresight research confirmed that ontology-aided solutions are much welcome by industries, which clearly exhibit a comprehensive pattern of needs and requirements that fir well to the existing or potential capacities of the ontology engineering. The outcomes from performed research can be exploited by further R\&D effort in a threefold way: (i) translation to core, domain and application ontologies, to support particular solutions or functionalities; (ii) verification of fundamental abstractions and formalizations, then ontology languages, and further research along this line; (iii) R\&D of novel systemic solutions and functionalities, which are enabled by the existing and expected capacities of ontology engineering; this way a feedback for (i) and (ii) could be obtained. Using the research findings with regard to (i) and (ii), the following topics can be suggested as of major importance: (a) expressiveness of ontologies, consid- 
ering particular complexities, which are specific for the domain, which might have spatiotemporal or mereotopological nature; (b) theoretical (meta-mathematical) foundations for ontology languages; (c) methods and tools for ontologies, particularly in reference to abilities of distributed knowledge and intelligence (i.e. beyond querying). Another perspective for further research can be derived from requested functionalities and features of future solutions. They can be categorized as follows:

- Novel forms of networking, like non-hierarchical, segmented, collectivities, herds;

- Local, global, distributed, externalized intelligence;

- Novel foundations of planning and control, ranging from localized selfmanagement (pull-flows), through collective distributed self-coordination, up to large scale forms, including centralized;

- New functionalities driven by diagnostic or adaptive abilities or other intelligence;

- New forms of encapsulation, i.e. other than data encapsulation; e.g. encapsulation of demands, flows, operations etc.

- New types of structures and infrastructures, up to economic institutions.

Finally, the ideations presented in this paper could be a subject of further R\&D effort.

Acknowledgements. This work has been co-funded by the PSP 504/02105/1103/ 40.000105 grant of Warsaw University of Technology and by the EU ARTEMIS project no. 332946 "ESCOP - Embedded systems for Service-based Control of Open manufacturing and Process automation". The author thanks to partners of the projects.

\section{References}

1. Berners-Lee, T., Hendler, T., Lassila, O.: The Semantic Web. Scientific American, 2001/V, pp. 34-43 (2001)

2. Montreuil, B. Towards a Physical Internet: Meeting the Global Logistics Sustainability Grand challenge. In: CIRRELT-2001-03 Research Report (2011)

3. Sendler, U. (Ed.): Industrie 4.0 - Die Beherrschung industrieller Komplexität mit SysLM. Springer (2013)

4. Lee, E.: Cyber Physical Systems: Design Challenges. In: Proc. $11^{\text {th }}$ IEEE Symposium on Object Oriented Real-Time Distributed Computing (ISORC), pp. 363-369 (2011)

5. Chandrasekaran, R, Josephson, J.R., Benjamins, V.R.: What are ontologies, and why do we need them? IEEE Trans. on Intelligent Systems, 1999/January-February, 20-26 (1999)

6. Lobov, A., Ubis Lopez, F., Villasenor Herrera, V., Puttonen, J., Martinez Lastra, J.L.: Semantic Web Services Framework for Manufacturing Industries. In: Proceedings of the IEEE International Conference on Robotics and Biomimetics, pp. 2104-2108 (2009)

7. Strzelczak, S.: Ontology-Aided Management. Silesian University of Technology Series in Management, 73,pp. 619-630 (2014)

8. Garetti, M., Macchi, M.: The role and trends of modeling and simulation in managing manufacturing complexity. In: Proceedings of the $7^{\text {th }}$ IFAC Workshop on Intelligent Manufacturing Systems, pp. 1-8 (2003)

9. Grubic, T., Fan, I.-S.: Supply chain ontology: Review, analysis and synthesis. Computers in Industry, vol.61, pp. 776-786 (2010) 